\title{
PHOTOGRAPHY AS AN AID TO THE EXPLORATION OF NEW COUNTRIES
}

THE primary object of all exploration is to collect information, and if the information collected is to be of permanent use to other people, it must be recorded in such fashion that the records can be correctly interpreted by people other than the creator of those records.

A very large proportion of all the facts of interest and importance which fall under the observation of an explorer are visible facts which can be best recorded as pictures or diagrams. It rarely happens that mere verbal description alone can afford a mental picture at all comparable in the matter of accuracy to a good painting or drawing or'a good photograph, supplemented by sufficient notes. It is therefore certain that an explorer who aims at the production of important work should be provided with adequate means for making accurate pictures, and he should be experienced in the preparation of maps and diagrams.

Formerly travellers had to depend largely upon their skill with pencil and paint brush to collect pictorial illustrations for the text of their written reports, but since the advent of modern dry plate photography and the more recent half tone printing process of reproduction photographs are nearly everywhere supplanting hand-made pictures for illustrative purposes.

With scarely any exceptions all the best modern books of travel are more or less copiously illustrated from photographs, and in a general way the more fully they are illustrated the better they are appreciated.

The photographs used for illustration purposes have been mostly taken and intended to be used as sketches had been mostly used before. They have been mostly taken with the 
idea of getting pretty interesting pictures of things seen by the traveller without any special reference to measurements of any kind. A large majority of people who take photographs have never learnt the art of photographic mensuration, and it is highly probable that even in these days there are many photographers who have never given. any serious thought to this aspect of photographic utility, but the time will come when measurable records will to a large extent supplant the toy records of the past and present. People will want to know something about the heights and distances and depths of objects depicted.

The exploration of any country must always be incomplete until it has been surveyed.

The preparation of a map, if no good map exists already, or the filling in of omissions in existing maps will always be among the most important tasks which an explorer should set before him if he wishes to contribute effectively to the development of a new country. The quickest and best possible way to collect ill the necessary information for making a map of any new country is to prepare a sufficient series of photographs overlooking the country from a number of station points judiciously chosen with reference to the character of the country to be surveyed and the scale of map which it is desired to make.

Good survey photographs may be quite as pretty as, and they will always be very much more useful than the ordinary run of photographs with which readers of modern books of travel are familiar. As every experienced photographer will know, it may very well happen that photographs which are taken in the ordinary way for picture making purposes only may very often yield untrue impressions.

The photographs are not really untrue projections, but the conditions under which they have been obtained may have been such as to yield pictures which tend to convey false impressions of relative size and distance as well as light and shade. The old saying that photography cannot lie may lead to much misconception unless it is correctly interpreted. In the sense that the photographic plate has only recorded the actinic light and shade impressions received on its surface, this is true. The plate has recorded truthfully. It could not do otherwise, but the impression conveyed to any one looking at a print from that

22 Vol. 1 
photographic record may be altogether wrong. For example, suppose a camera is badly tilted when an exposure is made for taking a picture of a mountain range, the appearance of the range and all the peaks will be unnatural in the picture, just as would the appearance of a building if the camera were tilted up or down. Again, suppose a view embracing a large angle is condensed on a space of a few square inches. Whether this view is obtained by direct exposure in the camera or by subsequent reduction, the invariable effect will be to convey false impressions of exaggerated distance and smallness in the central regions, and exaggerated size and nearness towards the margin. If the tilted pictures could he looked at from the right spot while tilted at a corresponding angle to the original tilt, they would look right, or if the small wide angle picture could be examined through a lens opposite the middle of the picture and at the right distance, then that would look right, or if the small wide angle picture were made into a lantern slide and projected on a good flat screen, then it would look right to any one close up against the lantern lens. Suppose again that a patch were cut out of one of the corners of a wide angle picture and examined by itself-it would look wrong, and if it were projected by a lantern it would still look wrong if it were thrown on the central part of the screen, but if its position behind the lantern lens were shifted until its image fell upon that part of the screen where it would have fallen if the whole picture of which it formed a part had been projected in the ordinary way, then it would appear right from the position of the lantern lens. The above are only a few illustrations of the general proposition that photographic pictures may mislead as regards the outline and shape of bodies and as regards their size and distance.

Photographs originally obtained on film surfaces which were buckled at the time of exposure will always be bad and look wrong more particularly if the focal length of the lens was small and the buckling considerable. If it were possible to reproduce the exact buckled conditions and then examine the picture through a tiny aperture at exactly the right spot it might look right but in practice a badly buckled film with short focus lens gives a hopelessly bad inaccurate picture. Then as regards light and shade the ordinary tendency is for contrasts of light and 
shade to be exaggerated in a photograph and it is quite possible for the exaggeration to be so great as to lead to serious misapprehensions. On the other hand, in a very intense light with over exposure, or in a very subdued light with under exposure, marked differences of light and shade in the object may not be at all represented in the image. It will happen only rarely that the photographic picture will show contrasts of light and shade in exact proportion as the eye would see them. Among other considerations to be taken into account it must be remembered that different colours have different actinic values for the halogen salts of silver and unless tinted ortho-chromatic plates with appropriate colour filter screens are used there may be great inequalities of light and shade values in the picture corresponding to different shades of colour on different parts of the field of view. Altogether it is evident that either from careless manipulation or deliberate intention, photographic pictures may be so made as to mislead by the impressions they produce on the mind of a person looking at them. An explorer who intends to take photographs of new things and places for illustration purposes should first master the practical application of photography to the art of picture making by abundant practice on familiar objects within reach.

Some study and much practice are necessary before any one can become a proficient photographer for the most ordinary illustrative purposes. A little more study and a little more careful attention are necessary to secure accurate pictorial records of permanent value, but taken altogether the total amount of time and study and effort necessary to make a man a good photographer are not probably so great as would be required to make him a good illustrator with paint brush and pencil, and there is a much greater likelihood in most cases that an intelligent man will be able to become a proficient photographer than a really accomplished draftsman. Also there can be little doubt that the results obtainable by photography will be much more copious and of greater real permanent value for scientific and practical purposes than even the best artist's drawing.

It is not necessary in this article to attempt to explain the ordinary processes of photographic manipulation. There are many practical schools where adequate instruction can be 
gained in the everyday commonplace methods of procedure. An explorer should be intimately familiar with those methods and be competent to employ them to the best advantage but he ought to aim also at something better in the shape of indubitable accuracy in the results produced and the means for interpreting those results.

An accomplished photographer will have learnt by study and experimental practice how to obtain pictures which are sharply defined and full of detail with all sorts of shades of gradation of tone approximately corresponding to relative shadow values in the objects depicted. When he has learnt how to obtain good pictures he should certainly proceed to study the most elementary geometrical conditions which determine the relationship between the positions of points in the picture and points in the objects depicted, so that it may be possible to deduce the actual forms and dimensions of objects from photographs of those objects. The value of photographs for purposes of illustration and record is enormously increased if the photographs can be accurately interpreted to give exact information of concrete facts apart from mere sensual impressions of the light and shade. appearance of objects depicted. When a photograph is taken of printed matter or manuscript or of a picture every practical photographer will know that the camera should be set up so that the surface on which the image is to be photographically impressed shall be truly parallel to the surface to be copied. Under these conditions with a good lens and truly flat surfaces for both object and image it is well known that the image surface will be an exact scale reproduction of the object surface, all linear dimensions on object and image being simply proportional to the conjugate focal distances of the object and image respectively.

Photographic enlargements and reductions and life-size reproductions are continually being prepared under these conditions and the accuracy of the photographic reproduction is indisputable and measurement from the photographs extremely easy.

It is not so generally understood that every photograph taken with a good lens on any plane surface is a geometrically correct projection which can be correctly interpreted if the necessary 
data for interpretation are known. Every plane photograph is a perspective view of the objects depicted and can be interpreted as a perspective if the data for interpretation are given.

The camera should be so constructed as to give these data automatically on the face of the picture at the time when the camera exposure is made. Cameras so fitted are now gradually finding their way into use among expert surveyors and no doubt in time the use of surveying cameras will become very general if not universal.

The chief data required are:-

(1) The principal point. (2) The principal horizontal line. (3) The principal vertical line.

Of these the principal point or centre is all important If that point is not known or cannot be found the accurate interpretation of the picture is in a general way impossible.

When buildings or other artificially constructed bodies with regular geometrical outlines are the objects photographed it may be possible sometimes with more or less difficulty to deduce the principal data but with ordinary landscape views, this cannot be done unless the precaution has been taken to secure that the photographs shall bear definite marks on their faces to indicate the centre and traces of the two principal planes. A good surveying camera will be fitted with means for automatically recording the necessary information and the explorer should certainly possess a camera so fitted.

Then he wants to know (4) The orientation of his views, and (5) The station points from which the views have been taken. This information must be available when the picture is to be interpreted for map making purposes, and may be obtained from notes recorded in a note book when the pictures were taken, or better from automatic records on the pictures themselves such as can be secured by the use of a good modern surveying camera.

In these days most good cameras are provided with levels to indicate when the picture plane is vertical and for ordinary picture work when exact accuracy is not aimed at these levels serve their purpose but for all mapping and measuring purposes not only should there be accurate levels on the camera, but also convenient levelling arrangements, and a very rigid stand. An 
explorer should try to collect and record information with as much accuracy as possible, and not rest content with any standard of accuracy below the best which it is in his power to obtain. With the object of collecting as much accurate pictorial information as possible he should certainly start with reliable up to date apparatus which can be trusted to give accurate results without requiring too much detailed attention or too many supplementary notes. It is false economy and great waste of labour to take and use imperfect instruments when better can be had and in the case of survey photographs in particular there will be an immense saving of time and avoidance of risks of error and confusion from wrong or omitted entries if the apparatus records automatically the essential information required about the pictures. There have been some great improvements effected in surveying cameras in quite recent years so that the collection of standard pictures is not now a difficult matter. If the traveller can find time to study and practice the art of plotting from photographs he will find this a useful accomplishment, and in any case if he makes a good collection of survey pictures he or others can plot from them at leisure, and innumerable details can be picked out and plotted when they are wanted.

In selecting stations from which to take survey photographs it is generally best to choose spots overlooking the ground, as for example the tops of hills, or in towns the highest buildings but other stations may often with advantage be selected at different altitudes and at the bottoms of valleys. When it is desired to obtain series of contour lines the quickest and best plan is to select series of stations in ascending or descending order on a hill side or on several hills.

All points cut by the horizon at any station are at the same level as the station so that it is only necessary to plot on the plan points bisected by that line to obtain a contour. If this is done for a number of stations at different known altitudes a number of contour lines will be obtained of known altitudes corresponding to the stations.

When it is desired to fix positions on a map from photographs the best plan is to intersect direction lines to those points as seen from two or more stations. The principle of fixing by 
intersection of rays is exactly the same for photographs as for plane tabling in the field with the great advantage that it is more rapid and often more convenient.

The detailed method for plotting has been explained by the writer and others in various publications to which reference may be made by any reader who desires to practise mapping out from photographs. For making plans from landscape photographs, the plan of plotting intersections of direction rays is the simplest and most accurate and most generally applicable, but for constructing the plans and elevations of buildings it is sometimes more convenient to apply the principles and practice of perspective using photographs from two or more stations to check and control results.

As a general rule photographs as originally taken are two small for manipulative treatment as perspectives. They need to be enlarged several diameters at least when the difficulties will be of the ordinary character appertaining to the projection of ground plans and elevations from perspectives. If the photographer when he has to deal with buildings, will take care to obtain a fair proportion of full front and diagonal views this will often save trouble afterwards. In frontal views of flat surfaces all linear dimensions are exactly proportional in image and object. The geometric elevation and the perspective are the same. The scale is simply the inverse ratio of the perpendicular distance of the frontal plane from the lens to the focal distance of the picture.

The heights of points appearing in a photograph above or below the horizon line can either be determined by perspective methods and scale measurement or by simple computation of values in the formula $\mathrm{H}=\mathrm{D} \tan a$, when $\mathrm{H}$ is the height required to be found, $D$ the distance and $a$ the angle subtended at the station by a perpendicular from the point on the horizon plane.

The most modern surveying camera designed by the writer is fitted with cross hairs in the focal plane to trace the horizon and principal vertical lines which intersect at the centre or principal point.

It is provided also with an internal magnetic compass which records automatically the bearing of the principal vertical plane 
containing the axis through the centre and the station, and it is furnished with a tangent scale in the focal plane, which prints on the picture a scale of horizontal angles reduced to the plane of the horizon, and there are tablets in carriers for memoranda, whose shadowgraphs are printed as latent images on the sensitive plate at the same time as the rest of the picture. The accompanying pictures will help to illustrate many of the matters which have been discussed in the earlier part of this article. The views overlooking Trafalgar-square will be recognised by many readers, and to every one familiar with the principles of perspective it will be evident at once that those pictures are really perspectives. They are too small for exact use in the direct construction of ground plans and elevations from the pictures, but if they are enlarged sufficiently it will be found that all the known principles of perspective can be applied to them, so that all kinds of information which could be extracted from good perspective drawings can be obtained from the photographs enlarged to corresponding dimensions. The horizon and principal vertical lines can be seen running right across the pictures. The centre is of course the point where they intersect.

The curved scale at the top gives the magnetic bearing of the principal axis. The straight divided scale immediately below it is a scale of reduced horizontal angles. The writing in the rectangular spaces at the top right and left corners shows what memoranda relating to the picture were recorded by the operator for reproduction on the picture before the original photographic exposure was made.

The magnetic bearing of any point in one of these pictures from the spot where the camera stood when the picture was taken, can be obtained immediately by observing how far the image of that spot lies to the right or left of the principal vertical line, when the transverse (tangent) scale shows how many degrees of angle that distance corresponds to. If the point lies to the right the magnetic bearing is the sum of the compass and tangent scale readings, or if to the left then the difference. The focal length of the lens or the equivalent focus for any enlargement can be obtained by simple geometrical construction or computation from the tangent scale. 

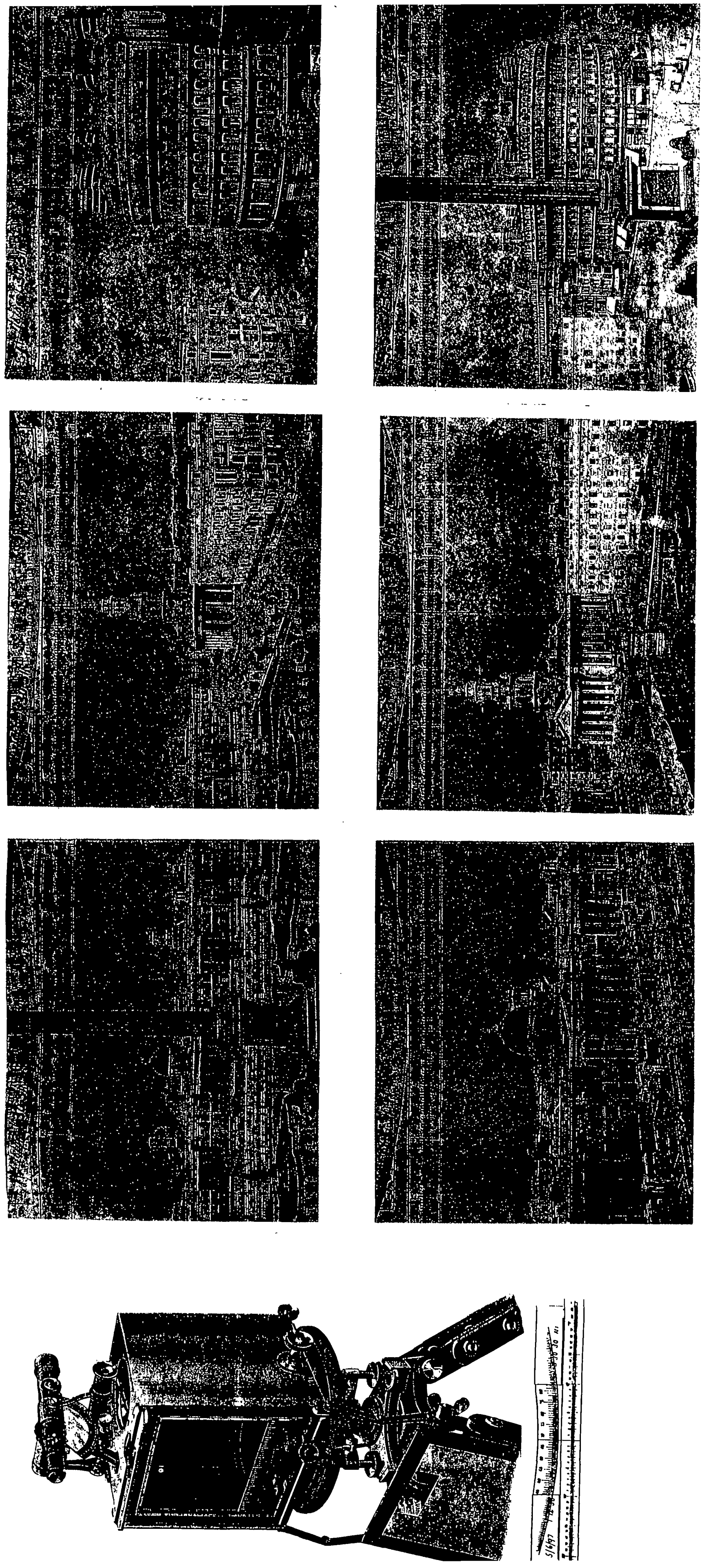

If the position of the station point on a plan is known then, knowing the focal length and the orientation it is easy to rule the trace of the picture plane on the plan, and so set off any number of direction lines from the station to any number of points whose images are visible in the pictures.

If views from two stations are available (such for example as the brace of views of part of Trafalgar Square from Drummond's Bank and the Union Club), then the intersections of direction lines from the two stations to any point will fix the position of that point on the plan.

Perspective methods which are generally applicable with advantage to the interpretation of buildings or other objects largely composed of straight lines, are not as a rule applicable to landscapes, except in special classes of cases. Lakes, bays, rivers, canals, and other approximately level surfaces may be plotted or measured by aid of superposed squares in perspective, but the method of plotting by intersection of rays is always available, and gives most accurate results.

The other figure is a photograph of the instrument with which some of these photographs have been taken.

J. BRIDGES LEE, M.A, F.G.S. 